Journal of Mathematics and Informatics

Vol. 18, 2020, 65-69

ISSN: 2349-0632 (P), 2349-0640 (online)

Published 12 February 2020

www.researchmathsci.org

DOI: http://dx.doi.org/10.22457/jmi.v18a6161

Journal of

Mathematics and

Informatics

\title{
Real Numbers with Fractional Presence
}

\author{
Hemanta K. Baruah \\ Department of Mathematics, The Assam Royal Global University \\ Guwahati - 781035, Assam, India \\ E-mail: hkbaruah@rgu.ac, hemanta@gauhati.ac.in
}

Received 10 January 2020; accepted 10 February 2020

\begin{abstract}
In the analysis of real numbers, the level of presence of an element in an interval is either 0 or 1 . In the mathematics of fuzziness, partial presence of elements with level of presence lying between 0 and 1 is studied. We are going to discuss regarding fractional presence of real numbers, the word fractional has been used here to mean that the level of presence can numerically be equal to any real number. It would be shown that the properties to define structures such as group and field are followed by real numbers with fractional presence. In this article, we are going to introduce the concept of negative partial presence that we shall use to define the operation of addition of fuzzy numbers $A$ and $(-A)$ from our standpoint.
\end{abstract}

Keywords: Real Numbers, Negative Presence

AMS Mathematics Subject Classification (2010): 03E72, 08A72

\section{Introduction}

In a subset of the real numbers, a number can be either present or absent. For example, in the set $A=[2,6]$, all real numbers from 2 to 6 would be included, and the level of presence of the numbers is 1 , and the number 7 for example is absent and therefore the level of presence of the number 7 in the set $\mathrm{A}$ is 0 . In this article, we are going to introduce the idea of fractional presence of an element in a set, where fraction would mean any real number. Indeed, we are basically aiming at introducing two things. First, we are going to introduce a concept of negative presence of real numbers. Secondly, we are going to show with an example that this concept of negative presence would be of use in the mathematics of fuzziness.

When an opaque object is placed in front of a small source of light, we see a shadow comprising of an umbra and a penumbra on a screen placed nearby. If we assume that the level of darkness in the umbra region is 1 , then the level of darkness in the penumbra region would be $1 / 2$. Accordingly, if a line segment is drawn through the center of the umbra, level of presence of darkness can be seen to be partial in the penumbra region. Therefore, fractional presence where the fraction is between 0 and 1 can indeed be visualized. Again, when we overwrite, the overwritten portion looks darker for multiple presence, and hence multiple presence can be visualized. 


\section{Hemanta K. Baruah}

In this article, we are going to discuss the case when the level of presence can be equal to any real number. We shall describe further that the real numbers with fractional presence follow the postulates needed to define structures such as Group and Field.

For the fractional presence that we are going to discuss, we indeed have nothing to refer to any earlier work done by any other author. For real numbers within an interval, partial presence with level of presence defined by membership values lying between 0 and 1, Zadeh [1] in 1965 introduced the theory of fuzzy sets. For the basic details, one may read the now classical books by Klir et al [2] and Zimmermann [3]. The theory of fuzzy sets has grown in various directions with applications in all sorts of fields. For some recent developments in theory and applications of fuzzy sets, we would like to cite Xiao and Xian [4], Zhang [5] and Yan and Yao [6]. In this article, we are going to introduce the concept of negative partial presence that we shall use to define the addition of fuzzy numbers $A$ and ( $-A$ ) from our standpoint.

\section{The idea behind}

If we superimpose a line segment over another, each covering a portion of the other, it can be seen that there would be a portion common to both of the line segments, and the common portion would look darker for double presence. If we now superimpose a third line segment over these two superimposed line segments such that it covers a part of the common portion with double presence, we would visualize triple presence too. The concept of superimposition of sets was introduced by the present author (see e.g. [7, 8, 9, 10]). In this example, we have described the level of presence of an element to be positive integers only. An extension of this idea can be to consider the presence level equal to a real number.

Let $a^{(v)}$ represent the real number $a$ with level of presence $v$. It can be seen that numerically, $a^{(v)}=v a, v \in R$, and $a \in R$. For example,

$$
4.0^{(2.54)}=2.54 \times 4.0=10.16=10.16^{(1.0)}
$$

and therefore 4.0 with level of presence equal to 2.54 is 10.16 with level of presence equal to 1.0. In the same way,

$$
\begin{aligned}
& 4^{(0.0)}=0.0, \\
& 4^{(-1.0)}=-4.0, \\
& 4^{(-2.54)}=-10.56=10.56^{(-1.0)} .
\end{aligned}
$$

Accordingly, for real numbers $a$ and $v$, it can be seen that $a^{(v)}$ can represent every real number. Therefore, for any given real number $a$ and for all real numbers $v$, the fractional number $a^{(v)}$ represents nothing but the set $R$ of the real numbers. Indeed, with 1 as base, $1^{(v)}=v$. Our standpoint is that the base can be just any real number. We are interested to see what $a^{(v)}$ represents when we consider at a time all $v \in R$, and all $a \in$ $R$.

Let us define a set $R^{(R)}$ comprised of all numbers $a^{(v)}, v \in R$, and $a \in R$. Before discussing the properties of the fractional numbers of the set $R^{(R)}$ we need to note the following:

(A) By definition, $a^{(v)}=v^{(a)}$

(B) For any $b \in R, a^{(v)}=b^{\left(\frac{a v}{b}\right)}$, by definition. 


\section{Real Numbers with Fractional Presence}

\section{Properties of $R^{(R)}$}

It can be easily seen that $a^{(v)}, b^{(\beta)}, c^{(r)}$ for $v, \beta, \gamma \in R$, and $a, b, c \in R$, follow the following:

(i) $\quad a^{(v)}+b^{(\beta)}=(a+b)^{\left(\frac{v a+\beta b}{a+b}\right)}$,

(ii) $\quad a^{(v)}+\left(b^{(\beta)}+c^{(\gamma)}\right)=\left(a^{(v)}+b^{(\beta)}\right)+c^{\gamma}$,

(iii) $\quad a^{(v)}+a^{(0)}=a^{(v)}$,

(iv) $\quad a^{(v)}+a^{(-v)}=a^{(0)}$ and

(v) $\quad a^{(v)}+b^{(\beta)}=b^{(\beta)}+a^{(v)}$.

$a^{(0)}$ here is an identity element; it could have been $0^{(v)}$ as well. $a^{(0)}$ is an identity because there is an uncountably infinite number of zeros in $R^{(R)}$, one for every real number including $a$. In the same way, $0^{(v)}$ is also an identity element for every $v \in R$. Further, $a^{(-v)}$ is an additive inverse of $a^{(v)}$ because $a^{(v)}=b^{\left(\frac{a v}{b}\right)}$ implies that $b^{\left(\frac{-a v}{b}\right)}$ is also an additive inverse of $a^{(v)}$ and therefore there is an uncountably infinite number of additive inverses of $a^{(v)}$. Accordingly, $R^{(R)}$ follows the postulates to define a Commutative Group with reference to the operation of addition of real numbers. Additive identities and additive inverses are however not unique.

It can further be easily seen that $a^{(v)}, b^{(\beta)}, c^{(\gamma)}$ for $\nu, \beta, \gamma \in R$, and $a, b, c \in$ $R$, follow the following:

(i) $\quad a^{(v)} \times b^{(\beta)}=(a \times b)^{(v \beta)}$ by definition,

(ii) $\quad a^{(v)} \times\left(b^{(\beta)} \times c^{(\gamma)}\right)=\left(a^{(v)} \times b^{(\beta)}\right) \times c^{\gamma}$,

(iii) $\quad a^{(v)} \times a^{\left(\frac{1}{a}\right)}=a^{(v)}, a \neq 0$,

(vi) $\quad a^{(v)} \times \frac{1}{a}\left(\frac{1}{v}\right)=a^{\left(\frac{1}{a}\right)}, a \neq 0$, and

(vii) $\quad a^{(v)} \times b^{(\beta)}=b^{(\beta)} \times a^{(v)}$.

$a^{\left(\frac{1}{a}\right)}$ here is an identity element. $a^{\left(\frac{1}{a}\right)}$ is an identity because there are an uncountably infinite number of multiplicative identities of the type $b^{\left(\frac{1}{b}\right)}$, one for every real number $b$. Further, $\frac{1}{a}\left(\frac{1}{v}\right)$ is a multiplicative inverse of $a^{(v)}$ because $a^{(v)}=b^{\left(\frac{a v}{b}\right)}$ implies that multiplicative inverses are not unique. Accordingly, $R^{(R)}-\left\{R^{(0)}\right\}$ follows the postulates todefine a Commutative Group with reference to the operation of multiplication of real numbers. Multiplicative identities and multiplicative inverses are however not unique.

Finally, it can be seen that $a^{(v)}, b^{(\beta)}, c^{(\gamma)}$ for $\nu, \beta, \gamma \in R$, and $a, b, c \in R$, follow the following distributive laws:

(i) $\quad a^{(v)} \times\left(b^{(\beta)}+c^{(\gamma)}\right)=\left(a^{(v)} \times b^{(\beta)}\right)+\left(a^{(v)} \times c^{(\gamma)}\right)$,

(ii) $\quad\left(a^{(v)}+b^{(\beta)}\right) \times c^{(\gamma)}=a^{(v)} \times c^{(\gamma)}+b^{(\beta)} \times c^{(\gamma)}$.

Therefore as

(a) $\left(R^{(R)},+\right)$ is a commutative group,

(b) $\left(R^{(R)}-\left\{R^{(0)}\right\}, \times\right)$ is a commutative group, and

(c) the distributive laws hold,

$\left(R^{(R)},+, \times\right)$ is a Field. Thus, we have seen that $R^{(R)}$ follows the properties needed to define structures such as Group and Field. 


\section{Hemanta K. Baruah}

Indeed, as can be seen, properties of the fractionally present real numbers $R^{(R)}$ are similar to those of the set $R$ of real numbers. Therefore, if we describe vectors with elements from the set $R^{(R)}$, it would be found that a set $V$ of vectors with respect to the operation of vector addition $(+)$ would form a linear space over the field $\left(R^{(R)},+, \times\right)$. It would further follow that the vectors from this space $V\left(R^{(R)}\right)$ with respect to the vector operation of scalar product (.) would follow the four postulates necessary for a linear space to be a Euclidean space.

\section{An application of the concept of fractional presence}

We are now going to give an example to show how this concept of fractional presence of real numbers may be useful in the theory of fuzzy sets. Assume that $A=[a, b, c]$ is a normal fuzzy number with a membership function $\mu(x)$, for $x \in[a, b, c]$. Let $B=$ $(-A)=[-c,-b,-a]$.

As is well known, the membership function of $B$ can be found from $\mu(x), x \in$ $[a, b, c]$. Now, the definition of fractional presence and therefore the concept of negative presence of a real number would lead to observe that the fuzzy number $B=[-c,-b,-a]$ with the membership function derived from $\mu(x), x \in[a, b, c]$ can be seen to be the same as the fuzzy number $A=[a, b, c]$ with the membership function $(-\mu(x)), x \in[a, b, c]$.

Now, we have as per our definition of fractional presence

$$
x^{\mu(x)}+x^{(-\mu(x))}=x^{(0)} \text {, for all } x \in[a, b, c] .
$$

Therefore, the fuzzy number $A$ with the membership function $(-\mu(x)), x \in[a, b, c]$, added to the fuzzy number $A$ with the membership function $\mu(x), x \in[a, b, c]$, added point for point for every $x$, would give us the fuzzy number $A=[a, b, c]$ with membership equal to 0 for all $x$ concerned, and of course the number $A$ with level of presence 0 for all $x$ concerned is nothing but 0 itself as per our definition of fractional presence of real numbers. So, the sum of the fuzzy numbers $A$ and $(-A)$ is nothing but 0 if we look into the matters from our standpoint. It may be noted that the sum of the fuzzy numbers $A$ and $(-A)$ is not equal to 0 if we perform the operation of addition in the classical way.

\section{Conclusions and discussions}

We have seen that every real number can be expressed in terms of fractional presence with reference to any real number. We have put forward a theoretical extension of the real number system. It may perhaps have applications in other fields of mathematics as well. In the real number system, there is a unique additive identity and a unique multiplicative identity. In our case, as both the additive identity and the multiplicative identity can be expressed in terms of every real number, the inverses are not unique, and they can be expressed in a countably infinite number of ways. Indeed, the description regarding the structural properties of the set $R^{(R)}$ might look trivial. But we have included the description with a view to showing that $R^{(R)}$ does follow the same structural formalisms followed by the set $R$ of real numbers.

For computational matters, any real number can be used as the base with reference to which every other number can be expressed. As such the proposed extension of the real number system may perhaps be of use in information science related matters. Further, our definition of fractional presence may actually have some applications in the mathematics of fuzziness. 


\section{Real Numbers with Fractional Presence}

\section{REFERENCES}

1. L.A.Zadeh, Fuzzy sets, Information and Control, 8(3) (1965) $338-353$.

2. G.J.Klir, Ute H. St. Clair and Bo Yuan, Fuzzy Set Theory, Prentice Hall, 1997.

3. H.J.Zimmermann, Fuzzy Set Theory - and Its Applications, $4^{\text {th }}$ Edition, Kluwer Academic Publishers, 2001.

4. H.Xiao and S.Xian, A linear method based on retrieval to estimate missing fuzzy-number in a fuzzy set and its application, Journal of Mathematics and Informatics, 12 (2018) 11 21.

5. S.-j.Zhang, Application of fuzzy comprehensive evaluation in environmental quality assessment, Journal of Mathematics and Informatics, 13 (2018) 55 - 63.

6. H.-y.Yan and B.-x.Yao, $(\lambda, \mu)$ - Fuzzy ideal of $(\lambda, \mu)$ - fuzzy subring, Journal of Mathematics and Informatics, 17 (2019) $131-144$.

7. H.K.Baruah, Set superimposition and its application to the theory of fuzzy sets, Journal of the Assam Science Society, 40 (1) (1999) 25 - 31.

8. H.K.Baruah, In search of the root of fuzziness: the measure theoretic meaning of partial presence, Annals of Fuzzy Mathematics and Informatics, 2 (1) (2011) 57 - 68.

9. H.K.Baruah, The theory of fuzzy sets: beliefs and realities, Int. J. of Energy, Information and Communication, 2(2) (2011) 1 - 22.

10. H.K.Baruah, Construction of the membership function of a fuzzy number, ICIC Express Letters, 5(2) (2011) 545 - 549. 